\title{
L'auteure mise en scène et mise à nu. Sur les fausses confessions de La nouvelle pornographie Joëlle Papillon, Université de Toronto
}

Si l'on croit avec Gérard Genette (79-80) que la métalepse correspond au mouvement par lequel l'on franchit le cadre dans une représentation, soit pour en sortir, soit pour mieux y entrer, alors la narratrice du roman de Marie Nimier La nouvelle pornographie (2000) joue de la métalepse et de procédés textuels connexes afin de brouiller les niveaux de réel et de fiction et d'emmêler le vrai et le faux, pour se protéger à la fois de l'exposition pornographique et du dévoilement autobiographique. Ici, la métalepse ne s'installe pas dans des adresses au lecteur ou dans l'ostentation du pouvoir de l'auteur à manipuler ses personnages, mais plutôt dans le jeu de superposition des deux niveaux de fiction identifiés par Genette (26), soit le «niveau (prétendu) réel » et le « niveau (assumé comme) fictionnel ». Lorsque le fictionnel déborde dans le réel, le réel en sort contaminé et sa vraisemblance durement touchée puisque, comme l'explique encore Genette (23), la convention romanesque exige que l'on croit, au moins durant la lecture, que ce qui est raconté est réellement advenu - ce qui est parfois difficile dans $L a$ nouvelle pornographie.

Revenant à la maison, Marie, la narratrice, découvre une lettre que lui adresse personnellement M. Jolicœur, directeur d'une compagnie d'objets ménagers qui cherche à lui vendre une «table à repasser révolutionnaire ». Rien de plus banal, sauf que Marie est écrivaine et qu'elle a l'imagination fertile. À partir de la phrase conventionnelle qui indique que le directeur est " à [s]on entière disposition pour [lui] prouver sa reconnaissance, et la solidité de son matériel » (Nimier, Nouvelle 13), Marie se prend à rêver à cette promesse au conditionnel : «Je l'imaginai s'allongeant sur le vaste tableau chauffant, à titre de démonstration. Un de ses techniciens presserait sur la pédale, le bruit de la turbine [...] s'élèverait » (13-14), etc. Soudainement, toutefois, ce qu'elle imaginait a pris forme, et le directeur est bel et bien couché sur la table à repasser dans la cuisine de Marie; la séduction publicitaire est devenue une séduction sexuelle. Cette rupture de cadre, de l'imaginaire au «prétendu réel » de la fiction, est marquée par un passage abrupt au passé simple - « son sexe se dressa, je le pris dans ma main » (15) - puis, tout 
de suite après, au présent de l'indicatif : « il me dit de serrer un peu plus. C'est le directeur qui parle à présent » (15-16). De la rêverie éveillée, l'épisode de la rencontre avec le directeur est passé au monde tangible, ce dont Marie est la première surprise : «j'ai entendu sa voix, la voix du directeur en personne, il ne se contente plus de m'envoyer du courrier, il me parle, à moi, la sous-merde qui un jour lui a commandé un lot de torchons à trente francs » (16). Suit la relation d'une rencontre sexuelle entre Marie et le directeur, qui vante maintenant les possibilités érotiques de sa table, qui se termine sur une sortie de cadre non moins abrupte puisque, après l'orgasme, « je me retrouvai dans la cuisine, mon ordinateur en état de veille et mes notes éparpillées sur la table » (24). Marie a retrouvé le réel qui, l'espace d'un moment, lui semble plus déroutant que le monde fantastique qu'elle vient de quitter, et l'on comprend ce que l'on vient de lire pendant les quinze dernières pages lorsque Aline, la colocataire de Marie, lui demande : « Tu n'as toujours pas fini ta nouvelle? ? (24).

Dans ce livre étrange qu'est La nouvelle pornographie, nous avons affaire à une pornographe récalcitrante; alors que son contrat est de produire une pornographie au féminin (ce qu'elle tentait de faire avec la nouvelle autour de la table à repasser), Marie est gênée par un malaise important : elle n'aime pas la pornographie. Elle privilégie alors toutes sortes de stratégies discursives comme la parenthèse, la digression ou l'ellipse pour l'éviter, la piéger et finalement l'évacuer de son propre texte ${ }^{1}$. La pornographe en herbe doit tout de même se colleter à certains épisodes érotiques, et c'est là qu'elle joue de la métalepse afin de mieux embrouiller les choses. Pour éviter le simplisme de la pornographie standard, Marie travaille ses épisodes érotiques pour les compliquer, les rendre non seulement invraisemblables mais presque illisibles, tant les personnages et les situations se dédoublent et s'entremêlent, sautant d'un cadre à l'autre. Par exemple, Marie et Aline rédigent une nouvelle pornographique qui met en vedette un pompier ; cependant, à la lecture, il n'y en a pas une, mais trois. D'abord une nouvelle ratée, dictée par Aline, qui les met en scène toutes deux avec un pompier (74-77), nouvelle que nous ne lisons pas comme telle, mais que nous voyons s'écrire, puisque c'est ce qui est mis en scène ici avant tout : «l'écriture d'une nouvelle pornographique ». Ensuite, la narration change, d'homodiégétique elle devient hétérodiégétique, et l'on découvre le jeune Tom, futur pompier et promis à une carrière pornographique puisqu'il est spécifié qu'il était 
«monté comme un âne » (82); nous assistons donc aux premiers émois sexuels du jeune Tom qui ont lieu dans le passé et dans un cercle complètement masculin d'où Marie et Aline sont absentes, et Aline n'intervient qu'une seule fois dans le récit, afin de poser une question à Tom qui relance une nouvelle anecdote érotique (82-86). Enfin, la nouvelle pornographique avec le pompier repart une dernière fois, la focalisation homodiégétique sur Marie retrouvée, et elle met alors en scène Aline et Marie avec le pompier Tom (8696). Mais est-ce bien le même Tom qui est sorti de sa nouvelle ? Pour opérer la transition entre les deux Tom, la narratrice écrit : «Une bonne heure s'était écoulée depuis l'arrivée de celui que nous appellerions également Tom, pour qu'il ne se sente pas gêné à la lecture de ces lignes » (86), ce qui laisse présumer non seulement que les deux Tom sont différents, mais que le second renvoie à une personne réelle, alors que du premier il avait été dit qu'il sortait de l'imagination d'Aline (82). L'on sait que Marie a remis à son éditeur Gabriel la nouvelle avec le pompier, et que celle-ci parâtra dans le livre qui lui a été commandé - intitulé La nouvelle pornographie - mais de quelle version de la nouvelle est-il question? Ne serait-ce que par ces décalages, La nouvelle pornographie écrite au sein du roman ne peut pas coïncider avec celle que nous lisons, justement parce que Gabriel s'applique à simplifier l'écriture de Marie, à tailler les fils qui sortent en désordre de son imaginaire et qui la font déborder du cadre pornographique, exactement les fils qui partent dans tous les sens que nous retrouvons à toutes les pages de notre Nouvelle pornographie.

Dès la seconde page du roman, le nom de l'auteure fait son apparition par le biais du dépliant publicitaire de la société Jolicœur qui s'adresse à « Marie Nimier ». C'est donc interpellée par un prospectus que «Marie Nimier » entre dans son huitième roman, le premier à la voir transformée en personnage. Si l'intrusion du nom propre de l'auteure peut être comprise comme une affirmation de réalité - tout ceci est vrai, voyez, ça m'est arrivé à moi, Marie Nimier - un jeu complexe et tordu entre réalité et fiction est déployé dans La nouvelle pornographie, qui prétend concurremment que tout ce qui s'y passe est vrai et faux. L'épisode déjà mentionné qui suit la lecture du dépliant publicitaire, est si farfelu qu'il neutralise immédiatement le poids du nom véridique de l'auteure, puisqu'il est peu vraisemblable que le directeur de la compagnie d'objets ménagers se déplace au moment précis où sa cliente lit son dépliant afin de tester de façon érotique sa table à 
repasser avec elle, avant d'être rejoint semble-t-il magiquement par un technicien et la colocataire de Marie qui tous se mettent à s'amuser avec la fameuse table. Mais, dès que l'illusion de réel est dissipée par l'exagération humoristique de la scène, elle revient par la bande avec le constat que le roman n'a en fait jamais prétendu que cette scène loufoque s'était réellement passée : il n'a fait que semblant de la raconter, car l'épisode consistait en une nouvelle pornographique en quelque sorte extérieure à lui, puisqu'elle est rédigée par un personnage du niveau narratif principal, qui se nomme également Marie Nimier. En repoussant l'invraisemblance dans la nouvelle qui apparaît physiquement ou visuellement close puisqu'elle est mise en retrait du texte par un blanc et un saut de page, le reste du roman sort intact de cette incartade fantaisiste et, pour le moment, les deux niveaux de fiction assumée comme telle et de prétendu réel se trouvent rétablis. $L a$ nouvelle pornographie joue donc de trois Marie Nimier, car l'auteure extratextuelle s'y représente alors qu'elle tente d'écrire des nouvelles pornographiques à la première personne dont certaines sont reproduites dans le livre que nous lisons. La Marie Nimier du prospectus et de la table à repasser peut difficilement être considérée comme un indice de réel, en raison de son statut de fictivité au deuxième degré (elle est le personnage d'un personnage), statut qui est exposé plutôt que dissimulé. En fait, le nom sur le prospectus représente la seule occurrence du nom propre en entier puisque, par la suite, la narratrice ne sera interpellée que par son prénom - ce dont nous ne nous rendons pas compte, puisqu'il y a un «pacte onomastique » complet dès l'incipit. Cependant, si l'on retranche la nouvelle pornographique initiale pour ne garder que le niveau narratif principal, le pacte onomastique est beaucoup moins fort, puisqu'il n'y aura plus la moindre apparition de « Nimier » dans le reste du récit. L'association de Marie avec la Nimier extratextuelle demeure toutefois, étant donnée la présence de plusieurs biographèmes identifiables à l'auteure, tels son lieu de naissance, des références à la mort de son père lorsqu'elle était enfant ou à des romans précédents.

Mais, justement, ces allusions aux romans précédents de Nimier, que des lecteurs fidèles ne sauraient manquer de remarquer, ne fonctionnent pas toutes comme des indices de réel. Tandis que la référence à son roman autobiographique Sirène (1985) est explicite, puisque la narratrice explique que son amie lui a offert un briquet en forme de sirène parce que son premier roman s'ouvrait sur une tentative de suicide où une jeune femme 
se jetait dans la Seine afin de « rejoindre ses sæurs les sirènes » (Nimier, Nouvelle, 184), quatre autres références à des romans de Nimier passeraient inaperçues pour des lecteurs moins familiers de son œuvre. Dans chaque cas, il s'agit d'une réapparition onomastique, où quatre personnages de La nouvelle pornographie sont présentés comme des personnes réelles dans le niveau narratif principal - celui qui est dans son ensemble prétendu réel d'un roman qui prend des couleurs autobiographiques, alors que ces mêmes noms sont utilisés dans d'autres romans de Nimier pour désigner des personnages dont on n'a aucune raison de remettre en question la fictivité. La plus mineure d'entre les quatre est Cora, personnage principal du roman fantaisiste L'hypnotisme à la portée de tous (1992) où elle est une jeune fille délurée, qui fait deux brèves apparitions dans La nouvelle pornographie $(135,143)$ lorsqu'on apprend que c'était là le nom de la poupée préférée de Marie lorsqu'elle était enfant. Le second personnage à réapparaître transformé est Valérie Toss, sortie de Celui qui court derrière l'oiseau (1996) où elle était une amie lesbienne du héros-narrateur, que l'on retrouve transfigurée dans La nouvelle pornographie (145147, 177); elle n'est désormais plus lesbienne, son caractère est complètement différent, elle est enceinte, et Marie la présente comme une amie d'enfance qu'elle n'a jamais revue depuis, mais à qui elle téléphone soudainement pour l'interroger sur sa vie sexuelle alors qu'elle-même souffre d'une crise d'inspiration. Pour les lecteurs fidèles, cette transformation de personnages complètement fictifs en personnages au statut fictif plus ambigu et associés à l'enfance que subissent Cora et Valérie Toss, produit un effet double : est-ce que derrière la Cora de L'hypnotisme à la portée de tous et la Valérie Toss de Celui qui court derrière l'oiseau se cachaient déjà les Cora et Valérie Toss « réelles » de l'enfance de Nimier, ou est-ce que ces dernières incarnations sont tout aussi fictives que les premières ? Est-ce que Cora redevenue poupée et Valérie copine de lycée constituent des preuves de réel ou est-ce que leur transformation importante entre les deux romans n'est pas plutôt l'indice d'un mensonge et d'un jeu où c'est le lecteur qui se fait avoir?

Deux autres personnages secondaires de L'hypnotisme à la portée de tous réapparaissent transformés dans La nouvelle pornographie, dont Puech, le standardiste de l'entreprise de téléphone rose où travaille Cora à la fin de ses aventures, qui est devenu ici Jean Puech (79-81, 87), l'intermédiaire à qui doivent téléphoner Marie et Aline 
lorsqu'elles sollicitent les services d'une escorte masculine afin de ressourcer leur inspiration pornographique. Le jeu entre les deux Puech est minime, puisque tous deux sont liés à l'industrie du sexe et au téléphone, et qu'ils se révèlent, l'un comme l'autre, des alliés de l'héroïne. Le personnage dont la réincarnation est la plus importante, est placé au centre de La nouvelle pornographie : c'est l'éditeur Gabriel Tournon. Dans L'hypnotisme, l'apparition de Gabriel relève presque du conte de fées, puisqu'il vient arracher Cora à son téléphone rose en lui proposant de publier son roman (qui n'a rien d'érotique) dont elle lisait des extraits au téléphone en remplacement du discours sexuel qui était attendu d'elle. Son portrait, bien que schématique, ne recoupe pas celui du Gabriel de La nouvelle pornographie puisqu'ils ne semblent pas travailler pour le même genre de maison d'édition : alors que Cora s'apprête à publier dans la collection prestigieuse d'une maison d'édition familiale reconnue, Marie est approchée par un éditeur spécialiste de la chose érotique. Le cas de Puech et Gabriel Tournon est donc différent de celui de Cora et Valérie Toss : alors que ces dames apparaissent radicalement changées d'un roman à l'autre, ce qui semble une transgression de la vraisemblance ou de la cohérence de l'œuvre, ces messieurs ne sont pas fondamentalement transformés, et l'on pourrait penser que, sans être le même personnage qui revient d'un roman à l'autre, ils correspondent pour Nimier à un type : un éditeur, pour elle, serait un Gabriel Tournon, un peu comme un amant, pour Camille Laurens, est un Jacques, sans que ce soit toujours le même Jacques.

La question du double se trouve ainsi liée, chez Nimier, à la question du faux, ce qui est intéressant pour une étude de la métalepse puisque quand l'auteure, Marie - qui est là, nous le présumons pour « Marie Nimier »-, intervient dans le texte, il est difficile de déterminer à qui nous avons affaire : la «Marie Nimier » interpellée par le prospectus publicitaire est-elle bien l'auteure de La nouvelle pornographie que nous lisons, et celleci est-elle la même qui a précédemment écrit Sirène, L'hypnotisme à la portée de tous et Celui qui court derrière l'oiseau ? Il y a nécessairement décalage, et Genette (109-110) voyait même dans l'ambiguïté du pronom je qu'un narrateur utilise pour désigner son protagoniste un «opérateur de métalepse ». Pour la narratrice de La nouvelle pornographie, il est clair que d'user de son nom propre et d'écrire au je ne constitue pas en soi un «pacte de vérité » puisque, en commentant son usage des pronoms personnels, 
elle se fait la réflexion que le je ne lui sert pas à exprimer une sorte de vérité personnelle (comme dans les confessions sexuelles, par exemple) mais bien à se travestir :

Je reviens à cette idée d'écrire les nouvelles pornographiques en mon propre nom. Longtemps j'ai hésité à me mettre en scène de façon aussi explicite, mais il me semble aujourd'hui que c'était la seule manière d'aborder honnêtement la proposition de l'éditeur. Non que ma propension à me confesser eût gagné du terrain, du grand déballage je me sentais fort éloignée, mais pour une raison plus souterraine : il m'aurait été impossible de faire vivre à une autre, fût-elle imaginaire, les débordements que je m'infligeais en tant que narratrice. Je me mouillais [...], je prenais sur moi, et par conséquent, symboliquement, j'avais tous les droits. Voilà ce que je gagnais en payant de ma personne, de ma première personne : la liberté de me travestir, de m'inventer, de me remodeler à loisir sans culpabilité majeure et même avec une certaine jubilation (Nimier, Nouvelle 110).

Pour elle, le je n'est donc pas une contrainte à la vérité autobiographique, mais plutôt une licence d'invention. Une fois le travestissement annoncé et revendiqué, la narratrice s'est créé un voile : qu'elle dissimule ou non, ses « confessions » ne pourront dès lors être comprises que comme une mise en scène. Même nue, l'écrivaine s'avance voilée, et le marché qu'elle a conclu avec le lecteur en "payant» de sa première personne semble aller comme suit : j'expose une «Marie Nimier », mais je vous avertis qu'elle ne sera pas moi, comme Valérie Toss n'était pas Valérie Toss. Par là, la narratrice de La nouvelle pornographie souligne la façon dont elle manipule et donc contrôle son autoreprésentation.

Elle fait d'ailleurs preuve d'une manipulation semblable dans la manière dont elle traite la pornographie qu'elle veut à la fois mettre dans son texte et neutraliser, c'est-àdire que sa façon d'utiliser un je qui n'est pas vraiment moi pourrait être rapprochée de celle avec laquelle elle écrit de la pornographie qui n'en est pas vraiment et qui dissimule en fait une histoire sentimentale. Dans les deux cas, le décalage s'appuie sur un changement de cadre, sur une rupture entre fiction «fictive » et fiction « réelle » : l'on croit Tom inventé de toutes pièces, puis il se retrouve dans la chambre de Marie, comme le directeur de la société Jolicœur qui sort de son dépliant publicitaire pour venir séduire 
sa cliente dans sa propre cuisine, accompagné de la table à repasser qu'il essaie de lui vendre ; l'on croit que «Marie Nimier » se dévoile à travers le récit de ses fantasmes et de ses souvenirs, mais elle se travestit et se met en fiction plus qu'elle ne se met à nu. Il y a donc beaucoup de «cadres » dans La nouvelle pornographie, et l'on ne sait pas toujours quand on est en train d'y entrer ou d'en sortir. L'on ne peut pas, non plus, se fier à la narratrice puisqu'elle nous manipule en jouant de faux personnages récurrents et de masques qui portent son nom, mais dont on ne sait quel visage se cache derrière. Ici, la métalepse proprement dite s'inscrit donc dans un contexte plus large où les niveaux narratifs sont constamment mélangés, traversés par les personnages, et où la fiction utilise des stratégies pour inscrire le réel mais afin de mieux se révéler fictive et insaisissable, redonnant son sens fort au terme « autofiction».

\section{Bibliographie}

Genette, Gérard. Métalepse : De la figure à la fiction. Paris : Seuil, coll. « Poétique », 2004.

Nimier, Marie. Celui qui court derrière l'oiseau. Paris : Gallimard, 1996.

---. La nouvelle pornographie. Paris : Gallimard, coll. «Folio », [2000] 2002.

---. L’hypnotisme à la portée de tous. Paris : Gallimard, 1992.

---. Sirène. Paris : Gallimard, 1985.

Papillon, Joëlle. «Penser la pornographie à la première personne : Marie Nimier ou le $\mathrm{je}(\mathrm{u})$ dangereux. » Le roman français de l'extrême contemporain : écritures, engagements, énonciations. Barbara Havercroft, Pascal Michelucci et Pascal Riendeau (dir.). Québec : Nota bene, coll. «Contemporanéités », 2010. 203-220.

\footnotetext{
${ }^{1}$ A ce sujet, consulter Papillon (2010).
} 